\title{
Metástasis cutáneas como primera manifestación de neuroblastoma primario de glándula suprarrenal en un paciente pediátrico
}

\author{
Abraham González-Mondragón ${ }^{1}$, Adriana M. Valencia-Herrera ${ }^{1 *}$, Mirna Toledo-Bahena1, \\ Carlos Mena-Cedillos ${ }^{1}$, Antonio Zamora-Chávez ${ }^{2}$ y Guillermo Ramón-García ${ }^{3}$ \\ ${ }^{1}$ Servicio de Dermatología Pediátrica; ${ }^{2}$ Departamento de Medicina Interna; ${ }^{3}$ Servicio de Patología. Hospital Infantil de México Federico Gómez, \\ Ciudad de México, México
}

\begin{abstract}
Resumen
Introducción: El neuroblastoma es el tumor maligno más frecuente en el primer año de vida y el tumor sólido extracraneal más frecuente en la infancia. Solo el 1\% de los casos debuta con metástasis cutáneas, caracterizadas por nódulos azulados subcutáneos. Se presenta el caso de un lactante con un neuroblastoma suprarrenal izquierdo en el que las metástasis cutáneas constituían el síntoma principal. Caso clínico: Lactante de sexo femenino, de 2 meses de edad, sin antecedentes de importancia para el padecimiento actual. Acudió por presentar dermatosis diseminada en la región cervical y occipital, el abdomen, el muslo derecho y el pie izquierdo. La dermatosis se caracteriza por nódulos subcutáneos, sólidos, bien delimitados, $<1 \mathrm{~cm}$, de color azulado, que iniciaron su aparición a los 7 días de vida en el hipocondrio derecho, con crecimiento progresivo, asintomáticos. Se realizó biopsia de un nódulo y se reportó la presencia de células pequeñas con núcleo denso hipercromático, escaso citoplasma y dispuestas en nidos. La inmunohistoquímica fue positiva para cromogranina y enolasa neuronal especifica. Los hallazgos fueron compatibles con metástasis cutánea de neuroblastoma. Se solicitó valoración y abordaje por oncología pediátrica, que reportó un estadio 4 de la enfermedad y se inició el tratamiento correspondiente. Conclusiones: Los pediatras y los dermatólogos pediatras son los primeros en atender a niños con alguna lesión cutánea. Se deben tener en cuenta las metástasis cutáneas, que pueden aparecer antes o simultáneamente al diagnóstico de un tumor primario. Por lo tanto, se debe realizar un correcto abordaje con el fin de mejorar el pronóstico y la calidad de vida del paciente.
\end{abstract}

Palabras clave: Síndrome blueberry muffin baby. Neuroblastoma. Metástasis cutáneas.

\section{Cutaneous metastasis as the first manifestation of primary adrenal gland neuroblastoma in a pediatric patient}

\section{Abstract}

Background: Neuroblastoma is the most common malignant tumor in the first year of life and the most common extracranial solid tumor in childhood. Only $1 \%$ of cases present with cutaneous metastases characterized by subcutaneous bluish nodules. We report the case of an infant with a left adrenal neuroblastoma in whom skin metastases were the main symptom. Case report: Two-month-old female infant with no relevant history for the current condition. The infant presented

Correspondencia:

*Adriana María Valencia Herrera

E-mail: adrianavalenciaherrera@gmail.com
Available online: 27-07-2021

Fecha de recepción: 16-10-2020 Fecha de aceptación: 06-12-2020 DOI: 10.24875/BMHIM.20000322
Bol Med Hosp Infant Mex. 2021;78(5):479-484

www.bmhim.com 1665-1146/@ 2020 Hospital Infantil de México Federico Gómez. Published by Permanyer. This is an open access article under the CC BY-NC-ND license (http://creativecommons.org/licenses/by-nc-nd/4.0/). 
disseminated dermatosis affecting the head in the cervical and occipital region, abdomen, right thigh and left foot. Dermatosis was characterized by subcutaneous nodules, solid, well limited, $<1 \mathrm{~cm}$, bluish color that appeared at 7 days of life in the right upper quadrant, with progressive growth, asymptomatic. A biopsy of a nodule was performed, which reported the presence of small cells with a dense hyperchromatic nucleus, scarce cytoplasm, arranged in nests. Immunohistochemistry was positive for chromogranin and specific neuronal enolase. Findings were consistent with cutaneous neuroblastoma metastasis. An assessment and approach by pediatric oncology were requested, reporting disease stage 4 and initiating the corresponding treatment. Conclusions: Pediatricians and pediatric dermatologists are the first to attend to children with a skin lesion. We must consider that skin metastases may appear prior to or simultaneously with the diagnosis of a primary tumor. Therefore, we should carry out a correct approach in order to improve the prognosis and the quality of life of the patient.

Keywords: Blueberry muffin baby syndrome. Neuroblastoma. Cutaneous metastases.

\section{Introducción}

El neuroblastoma es el tumor maligno más frecuente en el primer año de vida y el tumor sólido extracraneal más frecuente en la infancia ${ }^{1,2}$. El diagnóstico clínico a menudo es difícil, ya que el tumor primario permanece en un segundo plano y los síntomas están determinados por las metástasis. Se sabe que el $1 \%$ de los casos debuta con metástasis cutáneas caracterizadas por nódulos azulados subcutáneos ${ }^{3,4}$.

Se presenta el caso de una lactante con un neuroblastoma suprarrenal izquierdo en el que las metástasis cutáneas constituían el síntoma principal.

\section{Caso clínico}

Lactante de sexo femenino de 2 meses de edad, originaria del Estado de México, sin antecedentes de importancia para el padecimiento actual. Producto de la gesta III obtenido por parto, embarazo normoevolutivo, a término, Apgar de 8/9, peso de $3000 \mathrm{~g}$ y talla de $49 \mathrm{~cm}$. VDRL (Venereal Disease Research Laboratory) y virus de la inmunodeficiencia humana negativos.

Acudió a consulta por presentar dermatosis diseminada en la cabeza, el tronco y las cuatro extremidades, afectando la cabeza en la región cervical y occipital, el abdomen, el muslo derecho y el pie izquierdo. La dermatosis se caracterizaba por nódulos subcutáneos, sólidos, bien limitados, $<1 \mathrm{~cm}$, de color azulado, que aparecieron en el hipocondrio derecho a los 7 días de vida, con incremento progresivo en número y tamaño, asintomáticos (Figura 1). El resto de la exploración fue normal. Se realizó biopsia de uno de los nódulos, que reportó piel con hiperqueratosis ortoqueratósica y leve infiltrado linfocitario en la dermis superficial. Se encontró un nódulo subcutáneo compuesto por células pequeñas de tamaño uniforme, con núcleo denso hipercromático y escaso citoplasma, dispuestas en nidos
(Figura 2 A). La inmunohistoquímica resultó positiva para cromogranina y enolasa neuronal específica (Figura $2 \mathrm{~B}$ ), hallazgos compatibles con metástasis cutánea de neuroblastoma.

Se solicitaron valoración y manejo multidisciplinario por el servicio de oncología pediátrica, donde realizaron una tomografía toracoabdominal. Se encontró un tumor en la región superoanterior del riñón izquierdo, con dimensiones de $35 \times 49 \times 4 \mathrm{~mm}$. Las catecolaminas en orina (ácido vanilmandélico y ácido homovanílico) estaban elevadas. La biopsia suprarrenal guiada por ultrasonido reportó neuroblastoma poco diferenciado. El gammagrama óseo con metayodobencilguanidina (MIGB) mostró tejido captante en el cráneo, la región cervical, el tercio proximal de ambos húmeros, la pelvis, los fémures y las tibias. El aspirado de médula ósea reveló infiltración con el $6 \%$ de blastos. Se inició quimioterapia neoadyuvante con ciclofosfamida, doxorubicina y cisplatino. Posteriormente se realizó la resección quirúrgica del tumor residual $(34 \times$ $15.27 \mathrm{~mm}$ ) y se observó una aparente buena evolución. Sin embargo, 4 meses después la paciente presentó de nuevo nódulos subcutáneos. La biopsia de una lesión se reportó histológicamente como neuroblastoma bien diferenciado. La nueva gammagrafía con MIGB mostró una recaída en la parrilla costal, por lo que se inició el protocolo de tratamiento para recaída de neuroblastoma. Actualmente, la paciente se encuentra en seguimiento y tratamiento por oncología pediátrica.

\section{Discusión}

En 1863, Virchow describió por primera vez el neuroblastoma, un tumor derivado de las células de la cresta neural de la médula de la glándula suprarrenal o de los ganglios simpáticos ${ }^{2,5}$. La edad promedio al diagnóstico es de 21 meses a 2 años ${ }^{1,5}$. En México, en 


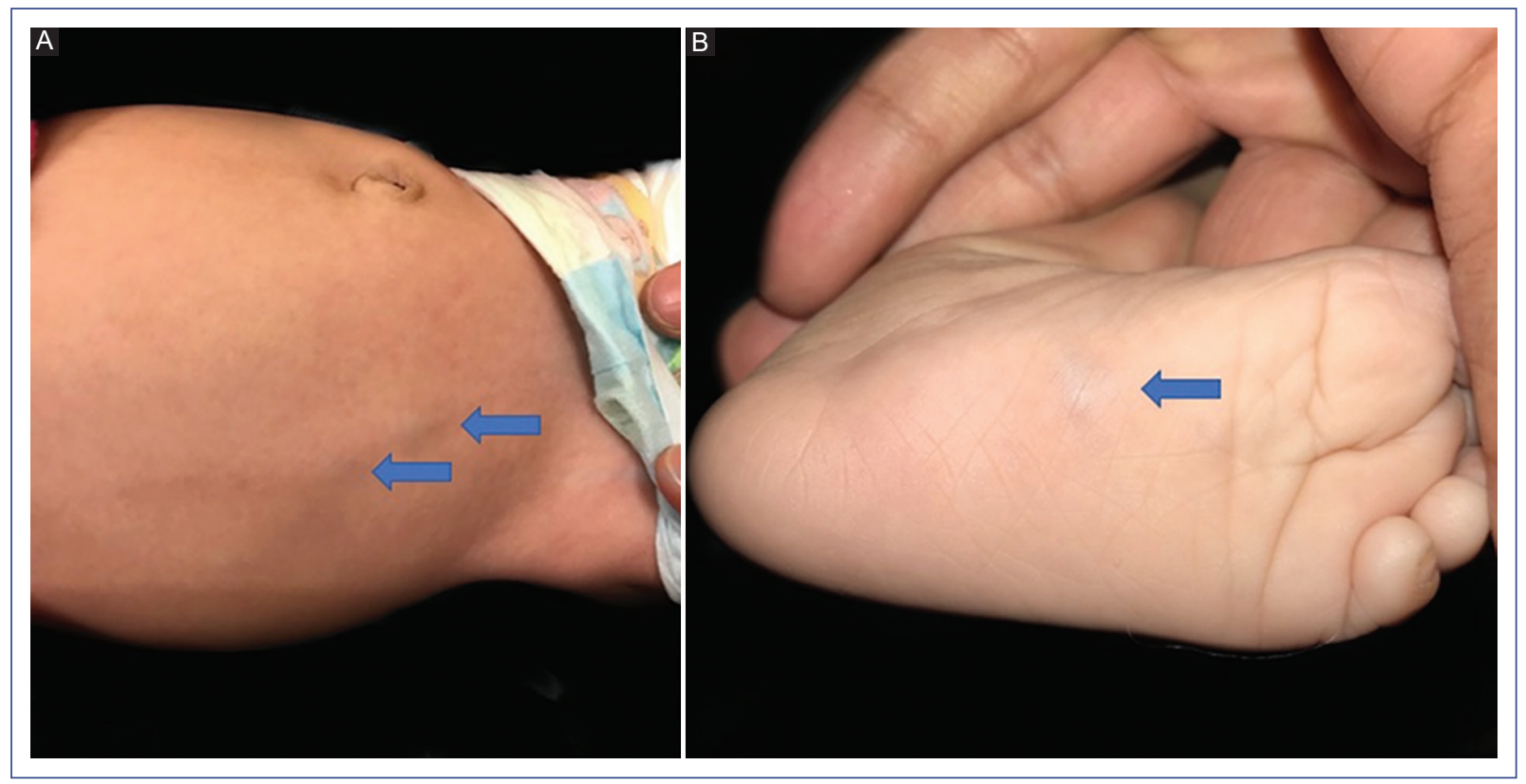

Figura 1. Nódulos subcutáneos en el abdomen (A) y la planta del pie izquierdo (B).

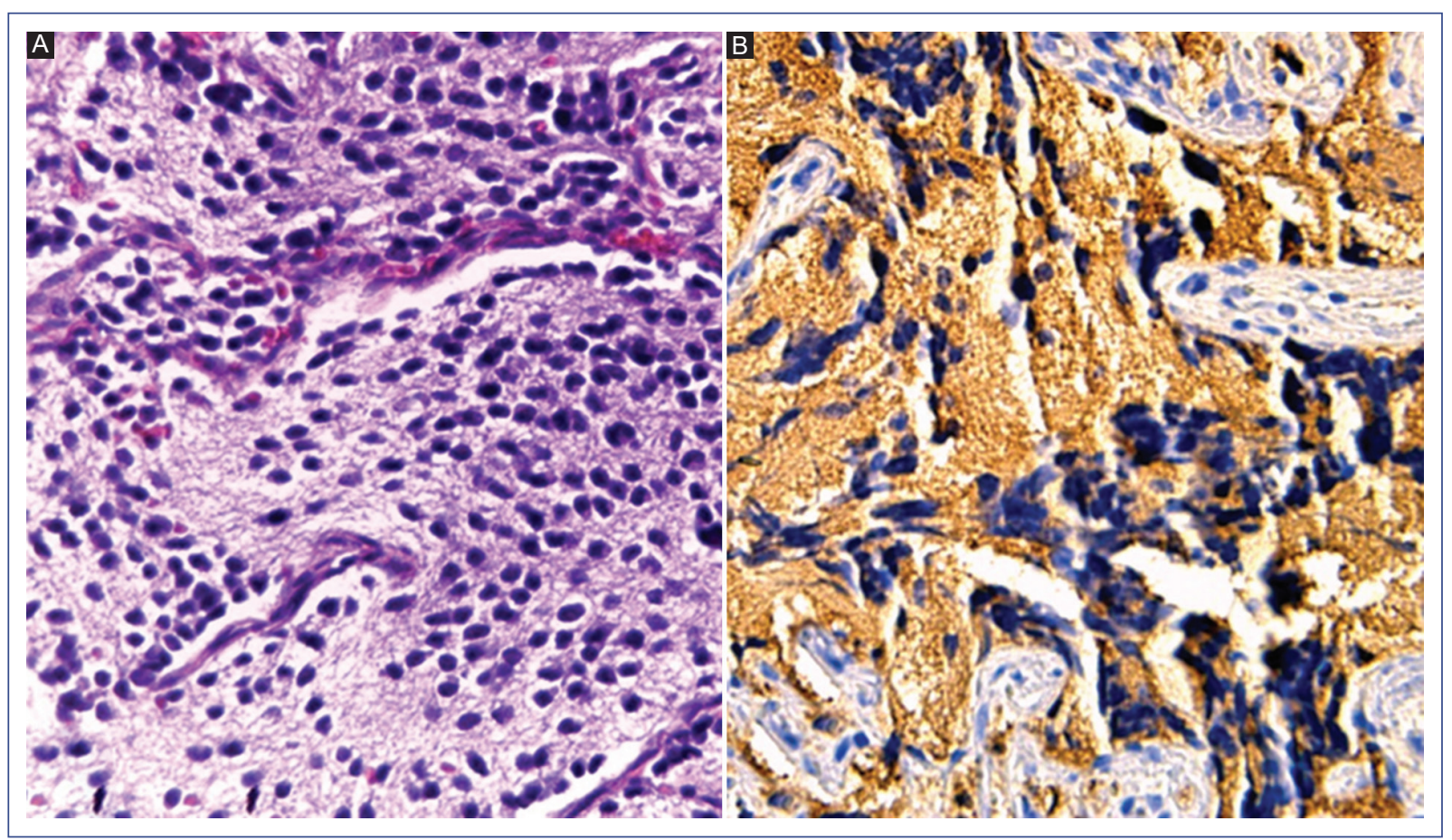

Figura 2. A: corte histológico de nódulo subcutáneo con células pequeñas de núcleo denso hipercromático y escaso citoplasma dispuestas en nidos. B: inmunohistoquímica positiva para enolasa neuronal específica.

2009 se registraron 2252 casos de cáncer pediátrico, de los cuales el $2.3 \%$ correspondieron a neuroblastomas, situándose en el décimo lugar en frecuencia en esta población ${ }^{4}$.
Algunos factores de riesgo se han asociado a su etiología, como el consumo de alcohol o drogas durante el embarazo, anemia materna, hipertensión gestacional, distrés respiratorio neonatal y Apgar $<7$ en el primer minuto de vida?. 
Tabla 1. Diagnóstico diferencial del blueberry muffin baby

- Eritropoyesis extramedular
- Infección congénita: virus Coxsackie B2, rubeola o parvovirus
B19
- Enfermedad hemolítica del recién nacido
- Síndrome de transfusión gemelo-gemelo
- Esferocitosis hereditaria
- Infiltración neoplásica
- Leucemia
- Histiocitosis de células de Langerhans
- Neuroblastoma
- Rabdomiosarcoma

Aunque la mayoría de los casos son esporádicos, el $1-2 \%$ presenta mutaciones en la cinasa del linfoma anaplásico (ALK) que se transmiten de forma autosómica dominante ${ }^{1,2}$. La presentación más común es una masa abdominal que se localiza en la glándula suprarrenal en el $65 \%$ de los pacientes ${ }^{6,7}$. Otros sitios de presentación son el cuello, el tórax, la pelvis y, con menor frecuencia, el timo, el pulmón, el riñón y el mediastino $0^{5,8}$.

El $1 \%$ de los casos pueden debutar con nódulos subcutáneos azulados, firmes, no dolorosos, móviles, de tamaño pequeño (de milímetros a centímetros), que dan al paciente la apariencia de un muffin de arándanos (blueberry muffin baby) ${ }^{4,7,8}$. Se localizan con mayor frecuencia en la cabeza y el cuello ${ }^{9}$. Aunque se originan por metástasis de la enfermedad, se asocian con buen pronóstico ${ }^{10,11}$. Algunos autores refieren que estos nódulos muestran un blanqueamiento característico, con un borde circundante de eritema cuando se palpan. El blanqueamiento dura de 30 a 60 minutos y se produce por liberación local de catecolaminas ${ }^{12}$. El $70 \%$ de los casos de infiltración a la piel ocurren en neonatos o lactantes menores de 3 meses. Las lesiones mejoran con el tratamiento hasta en el $80 \%$ de estos pacientes, y el resto presentan regresión espontánea ${ }^{11,13}$.

Ante un paciente con lesiones de blueberry muffin, el diagnóstico diferencial se debe realizar con patologías que condicionen eritropoyesis extramedular o infiltración neoplásica (Tabla 1) 13,14. La evaluación se inicia con la revisión de los antecedentes perinatales y estudios de laboratorio con enfoque en las serologías infecciosas en la madre y el neonato. Los hallazgos en la exploración física y las pruebas de laboratorio normarán la conducta para continuar el abordaje, ya que las lesiones cutáneas son similares en todas las patologías $^{15}$. Las infecciones virales producen anemia crónica en los fetos y estimulan la eritropoyesis dérmica en respuesta a la mayor demanda de glóbulos rojos ${ }^{14}$. Del complejo TORCH (toxoplasma, otras infecciones, rubeola, citomegalovirus y herpes), las más frecuentes son la infección congénita por rubeola y el citomegalovirus; los pacientes presentan prematuridad y peso bajo para la edad gestacional, además de cursar con sordera, coriorretinitis, hepatoesplenomegalia y retraso psicomotor ${ }^{16}$.

Si se sospecha que la etiología corresponde a alteraciones hematológicas, se deben realizar una biometría hemática completa y frotis periférico ${ }^{15}$. Las más frecuentes alteraciones son la enfermedad hemolítica del recién nacido y la esferocitosis hereditaria, que pueden presentarse con edema generalizado, anemia hemolítica, Coombs positivo e hiperbilirrubinemia no conjugada. El síndrome de transfusión de gemelo a gemelo puede ocurrir en cualquier embarazo gemelar monocoriónico; por lo general es benigno, pero cuando es grave, el gemelo donante presenta bajo peso al nacer, anemia e insuficiencia cardiaca ${ }^{16}$.

En las patologías neoplásicas, las lesiones cutáneas son provocadas por metástasis a la piel, por lo que es importante la biopsia de la lesión para el análisis por inmunohistoquímica y el aspirado de médula ósea. Entre las más frecuentes se encuentran la leucemia mieloide aguda y la leucemia congénita, que pueden manifestarse con infiltración cutánea, leucemia cutis, semanas o meses antes de la infiltración sistémica, anunciando un mal pronóstico ${ }^{12}$. Estos pacientes presentan además palidez, letargo, leucocitosis, fiebre y alteraciones neurológicas. La histiocitosis de células de Langerhans puede afectar la piel en más del $50 \%$ de los pacientes; en esta enfermedad se agregan pápulas y nódulos de color marrón-amarillento y con una ulceración central, además de dermatitis seborreica y múltiples petequias ${ }^{16}$. El rabdomiosarcoma en la etapa neonatal es raro y las metástasis a la piel son, por lo general, lesiones únicas de predominio en la cabeza $^{12}$. Shih, et al. ${ }^{14}$ reportaron un caso de blueberry muffin baby en el que coexistían lesiones de eritropoyesis dérmica y de neuroblastoma metastásico. El neuroblastoma puede presentar metástasis a otros órganos, como las glándulas parótidas, los pulmones, los huesos, la médula espinal, el hígado y la órbita, condicionando hepatomegalia, proptosis y equimosis en estos últimos dos sitios, respectivamente ${ }^{12,16,17}$. 
El diagnóstico se complementa con ultrasonido, tomografía o resonancia magnética para precisar las relaciones anatómicas con los troncos vasculares circundantes y descartar la invasión del canal medular. En el $85 \%$ de los casos, los metabolitos de las catecolaminas urinarias (ácido vanilmandélico y ácido homovanílico) se encuentran elevados ${ }^{2,8,16}$.

Para el diagnóstico definitivo se requiere un estudio anatomopatológico. El neuroblastoma se muestra como un tumor de células azules, redondas y pequeñas, de tamaño uniforme, con núcleo denso hipercromático y escaso citoplasma. La presencia de prolongaciones neuríticas es patognomónica (pseudorrosetas de Homer-Wright) ${ }^{1,6}$. Por microscopía electrónica se pueden observar gránulos neurosecretores y neurotúbulos ${ }^{10,12}$. Cuando estos hallazgos no son evidentes, se puede solicitar inmunohistoquímica con los siguientes anticuerpos: CD56, OM-1, NK-1, CD10, enolasa neuronal específica, cromogranina, sinaptofisina, HepPar-1, PGP 9.5 y EMA ${ }^{5,12,18}$.

El Sistema Internacional de Estadificación del Neuroblastoma (INSS) clasifica la enfermedad según sus características clínicas, radiológicas y quirúrgi$\operatorname{cas}^{5,19}$. El rango de la escala es de $1 \mathrm{a} 4 \mathrm{~S}$, y se correlaciona con el pronóstico ${ }^{5,20}$. El estadio 4 involucra un tumor metastásico a diferentes órganos (piel y huesos), los ganglios linfáticos o la médula ósea, y se considera de alto riesgo $0^{1,21}$. Según la cantidad de estroma, la diferenciación y los índices de mitosis y cariorrexis, se ha clasificado en tres categorías: indiferenciado, poco diferenciado y diferenciado ${ }^{1,22}$. Por último, el Sistema Internacional de Estadificación del Grupo de Riesgo de Neuroblastoma (INRGSS) lo clasifica en cuatro estadios (L1, L2, M y MS) de acuerdo con la presencia de 13 factores, incluyendo el estadio INSS, la diferenciación del tumor y las características que otorgan un pronóstico desfavorable: amplificación del gen N-MYC, pérdida del cromosoma 11q, 1p y 14q, ganancia del cromosoma 17q, diploidía y edad menor de 2 meses en el momento del diagnóstico ${ }^{12,20,23}$. El estadio M corresponde a la enfermedad diseminada en el momento del diagnóstico ${ }^{21,24}$. La paciente del presente caso no contaba aún con el estudio genético, por lo que se clasificó de acuerdo con el INSS como estadio 4 (enfermedad metastásica).

El tratamiento se basa en los esquemas establecidos por el grupo internacional de neuroblastoma SIOPEurope (SIOPEN) o por el Grupo de Oncología Infantil $(\mathrm{COG})^{25,26}$. Para los pacientes con neuroblastoma de riesgo bajo e intermedio (estadios 1 o 2 y $4 S$ sin amplificación del gen $N-M Y C$ ) puede realizarse la resección quirúrgica o mantenerse en observación ${ }^{27}$. Se han reportado tasas de sobrevida general del $99 \%$ para los pacientes en estadio 1 y del $93 \%$ para los pacientes en estadio $2^{26,27}$.

El 7-10\% de los neuroblastomas neonatales son de estadio 4S, con masas suprarrenales pequeñas y $\sin$ metástasis. Solo se mantienen bajo observación, ya que el $90 \%$ de estos pacientes cursan con buen pronóstico y regresión espontánea $8,20,28$.

En cambio, para la enfermedad de riesgo intermedio (estadios 2 y 3 con histología favorable) se agregan dosis moderadas de quimioterapia y la resección quirúrgica, y se reportan tasas de sobrevida general del $96 \%$ a los 3 años $^{27}$.

En los casos de alto riesgo (estadios 4 y 3 con histología desfavorable), como esta paciente, las tasas de supervivencia son del 40-50\%. Los regímenes de quimioterapia comprenden lo siguiente: inducción, combinando antraciclinas, compuestos con platino, agentes alquilantes e inhibidores de la topoisomerasa II (topotecán, ciclofosfamida, etopósido, vincristina y doxorubicina); control local, con resección quirúrgica después de 4-6 ciclos de terapia de inducción y radiación al sitio primario o los sitios de enfermedad residual activa; consolidación, con quimioterapia mieloablativa a base de melfalán o busulfán, carboplatino y etopósido, y rescate de células madre autólogas; y mantenimiento, con la combinación de inmunoterapia (dinutuximab, un anticuerpo monoclonal quimérico anti-GD2), factor estimulante de colonias de granulocitos y macrófagos, interleucina-2 y ácido retinoico ${ }^{25-27}$.

Desafortunadamente, hasta el $20 \%$ de los pacientes presentan mala respuesta a la inducción o recidiva del neuroblastoma, como el caso aquí descrito. La sobrevida en estos casos se reduce, por lo que se deben utilizar protocolos de segunda línea con agentes inhibidores de la isomerasa combinados con ciclofosfamida, ifosfamida, etopósido, doxorubicina y carboplatino ${ }^{27}$. El $67 \%$ de las muertes se deben a recidiva de la neoplasia, y el resto, a complicaciones secundarias al tratamiento ${ }^{4}$.

En el Hospital Infantil de México Federico Gómez se han registrado 46 pacientes con diagnóstico de neuroblastoma en los últimos 5 años. El caso aquí descrito es el único registrado con metástasis a la piel. Aunque la mayoría de los pacientes con neuroblastoma metastásico tienen un pronóstico favorable, algunos pueden presentar una evolución insidiosa, con recaídas y deterioro de la calidad de vida, como el presente caso. Puesto que los 
pediatras y los dermatólogos pediatras son los primeros médicos en atender a los niños con alguna lesión cutánea, se deben considerar las metástasis cutáneas y su comportamiento variable, ya que estas pueden aparecer antes o simultáneamente con otras manifestaciones de la enfermedad. Resulta fundamental realizar un correcto abordaje para establecer un diagnóstico temprano, y de esta manera mejorar el pronóstico de estos pacientes.

\section{Responsabilidades éticas}

Protección de personas y animales. Los autores declaran que para esta investigación no se han realizado experimentos en seres humanos ni en animales.

Confidencialidad de los datos. Los autores declaran que han seguido los protocolos de su centro de trabajo sobre la publicación de datos de pacientes.

Derecho a la privacidad y consentimiento informado. Los autores han obtenido el consentimiento informado de los pacientes o individuos referidos en el artículo. Este documento obra en poder del autor de correspondencia.

\section{Conflicto de intereses}

Los autores declaran no tener ningún conflicto de intereses.

\section{Financiamiento}

Recursos propios.

\section{Agradecimientos}

A mi sobrina Jessy, mi familia y maestros.

\section{Bibliografía}

1. Barrena-Delfa S, Rubio-Aparicio P, Martínez-Martínez L. Neuroblastoma. Cir Pediatr. 2018:31:57-65.

2. Lakhoo K, Sowerbutts H. Neonatal tumours. Pediatr Surg Int. 2010;26:1159-68.

3. Fernández-Teijeiro-Álvarez A Galán-del-Río P, Quintero-Calcaño V, Montiano-Jorge JI, Astigarraga-Aguirre I, Navajas-Gutiérrez A. Nódulos subcutáneos como forma de presentación de síndrome linfoproliferativo maligno. An Pediatr (Barc). 2009;71:148-52.
4. Méndez-Flores S, Gatica-Torres M, Zamora-González A, Domínguez-Cherit J. Metástasis cutáneas. Med Int Mex. 2015;31:434-40.

5. Costa AD, Zerbini MCN, Cristofani L. Metastatic congenital neuroblastoma associated with in situ neuroblastoma: case report and review of literature. Autops Case Rep. 2014;4:27-33

6. Balaguer J, Castel V. Neuroblastoma. An Pediatr Contin. 2008:6:276-83.

7. Castillo CP. La piel como fuente de malignidad. Rev Chil Pediatr. 2020;72:466-72.

8. Kumar M, Batra G, Saun A, Singh R. Blueberry muffin baby: an unusual presentation of infantile neuroblastoma. Indian $\mathrm{J}$ Med Paediatr Oncol. 2018;39:263-5.

9. Choate EA, Nobori A, Worswick S. Cutaneous metastasis of internal tumors. Dermatol Clin. 2019;37:545-54.

10. Consejo de Salubridad General. Guía de Práctica Clínica. Diagnóstico y tratamiento del neuroblastoma en el paciente pediátrico. México: Secretaría de Salud; 2010.

11. Álvarez AM, Lamarre R. Estado del arte: tumores de cresta neural, neuroblastoma. Fisiopatología, diagnóstico y tratamiento. Revista Med. 2012;20:38-49.

12. Holland KE, Galbraith SS, Drolet BA. Neonatal violaceous skin lesions: expanding the differential of the "blueberry muffin baby". Adv Dermatol. 2005;21:153-92.

13. Isaacs $\mathrm{H}$ Jr. Cutaneous metastases in neonates: a review. Pediatr Dermatol. 2011;28:85-93.

14. Shih YL, Kuo TT, Weng YH, Tseng FW, Lin JY, Ho HC, et al. Coexisting metastatic neuroblastoma and dermal erythropoiesis in a blueberry muffin baby. J Am Acad Dermatol. 2011;64:1197-8.

15. Shah VH, Rambhia KD, Mukhi JI, Singh RP. Blueberry muffin baby with cytomegalovirus hepatitis. Indian Dermatology Online J. 2019;10:327-9.

16. Mehta V, Balachandran C, Lonikar V. Blueberry muffin baby: a pictorial differential diagnosis. Dermatol Online J. 2008;14:8.

17. Chew YK, Cheong JP, Brito-Mutunayagam S, Khir A, Prepageran N, Mun KS. Subcutaneous metastasis of olfactory neuroblastoma - an uncommon presentation. Med J Malaysia. 2011;66:62-3.

18. Abo-Elenain A, Naiem Y, Hamedhosam-Eldin H, Emam M, Elkashef W, Abdel Rafee A. Right adrenal gland neuroblastoma infiltrating the liver and mimicking mesenchymal hamartoma: a case report. Int J Surg Case Rep. 2015;12:95-8.

19. Gauchan E, Sharma P, Ghartimagar D, Ghosh A. Ganglioneuroblastoma in a newborn with multiple metastases: a case report. J Med Case Reports. 2017;11:239.

20. Taggart DR, London WB, Schmidt ML, DuBois SG, Monclair TF, Nakagawara A, et al. Prognostic value of the stage $4 S$ metastatic pattern and tumor biology in patients with metastatic neuroblastoma diagnosed between birth and 18 months of age. J Clin Oncol. 2011;29:4358-64.

21. Cohn SL, Pearson AD, London WB, Monclair T, Ambros PF, Brodeur GM, et al. The International Neuroblastoma Risk Group (INRG) classification system: an INRG Task Force report. J Clin Oncol. 2009;27:289-97.

22. Chatten J, Shimada H, Sather HN, Wong KY, Siegel SE, Hammond GD. Prognostic value of histopathology in advanced neuroblastoma: a report from the Childrens Cancer Study Group. Human Pathol. 1988; 19:1187-98.

23. Park JR, Eggert A, Caron H. Neuroblastoma: biology, prognosis, and treatment. Pediatr Clin North Am. 2008;55:97-120.

24. Monclair T, Brodeur GM, Ambros PF, Brisse HJ, Cecchetto G, Holmes K, et al. The International Neuroblastoma Risk Group (INRG) staging system: an INRG Task Force report. J Clin Oncol. 2009;27:298-303.

25. Valteau-Couanet D, Schleiermacher G, Sarnacki S, Pasqualini C. [High-risk neuroblastoma treatment strategy: The experience of the SIOPEN group]. Bull Cancer. 2018;105:918-24.

26. Swift CC, Eklund MJ, Kraveka JM, Alazraki AL. Updates in diagnosis, management, and treatment of neuroblastoma. Radiographics. 2018;38:566-80.

27. Whittle SB, Smith V, Doherty E, Zhao S, McCarty S, Zage PE. Overview and recent advances in the treatment of neuroblastoma. Expert Rev Anticancer Ther. 2017;27:369-86.

28. lehara T, Hiyama E, Tajiri T, Yoneda A, Hamazaki M, Fukuzawa M, et al. Is the prognosis of stage $4 \mathrm{~s}$ neuroblastoma in patients 12 months of age and older really excellent? Eur J Cancer. 2012;48:1707-12. 